Article

\title{
Low-Complexity Blind Selected Mapping Scheme for Peak-to-Average Power Ratio Reduction in Orthogonal Frequency-Division Multiplexing Systems
}

\author{
Yujie Xia ${ }^{1, *}$ and Jinwei $\mathrm{Ji}^{2}$ \\ 1 School of Physics and Electronic Information, Luoyang Normal University, Luoyang 471022, China \\ 2 The 54th Research Institute of China Electronics Technology Group Corporation, Shijiazhuang 050081, \\ China;ji_jinwei@163.com \\ * Correspondence: yjxia_2001@163.com; Tel.: +86-379-68618309
}

Received: 7 August 2018; Accepted: 29 August 2018; Published: 31 August 2018

\begin{abstract}
Orthogonal frequency-division multiplexing (OFDM) is an attractive multicarrier technique for the simplicity of equalization and high data throughput. However, the transmitted OFDM signal has a very high peak-to-average power ratio (PAPR), which severely degrades the performance of practical OFDM systems and reduces the efficiency of high-power amplifiers (HPA). The selected mapping (SLM) scheme is an effective PAPR reduction method of OFDM signals. However, this approach usually requires side information (SI) transmission, which increases the difficulty of the hardware implementation with high complexity and reduces the data transmission rate. In this paper, based on designing phase rotation vectors in the time domain, a novel blind SLM method with low complexity is proposed to reduce the PAPR of OFDM signals. At the transmitter, the proposed method properly designs the phase rotation vectors in the time domain, which can be considered as an equivalent wireless channel without SI transmission. At the receiver, the effect of phase rotation vectors can be removed by the conventional channel estimation method, and the data demodulation processing can be easily performed by the frequency domain equalization. Simulation results show that the proposed scheme can achieve low complexity in PAPR reduction and has great robustness in bit error rate (BER) performance compared to the other low-complexity SLM PAPR schemes.
\end{abstract}

Keywords: orthogonal frequency division multiplexing (OFDM); selected mapping (SLM); peak-to-average power ratio (PAPR); side information (SI)

\section{Introduction}

Orthogonal frequency-division multiplexing (OFDM) is an effective technique for multicarrier systems due to its advantages of simplicity of equalization, high-rate data transmission and robustness against multipath fading. Currently, OFDM has been widely adopted by many modern wireless communication systems such as digital video broadcasting (DVB), digital audio broadcasting (DAB), wireless local area networks (WLAN) IEEE 802.11a, the WiMax standard IEEE 802.16 and long-term evolution (LTE). However, a high peak-to-average power ratio (PAPR) is one of the major problems associated with OFDM multicarrier systems, which may cause a severe degradation of bit error rate (BER) when it passes through a high-power amplifier (HPA) resulting in nonlinear distortion of the OFDM signal [1].

To deal with the high PAPR problem of the transmitted OFDM signals, many PAPR reduction techniques have been proposed [2-5]. These techniques include clipping, coding, nonlinear companding transforms, constellation extension, partial transmit sequences (PTS) and selected mapping (SLM). Among these techniques, SLM is an effective PAPR reduction technique with 
high performance. However, the conventional SLM PAPR reduction scheme usually requires very high computational complexity. To reduce the computational complexity of the conventional SLM PAPR reduction scheme, many modified SLM PAPR reduction schemes have been proposed [6-10], most of which reduce the computational complexity of the conventional SLM PAPR reduction by decreasing the number of inverse fast Fourier transforms (IFFTs) involved in generating candidate signals. For instance, in [6], a modified SLM scheme was proposed to reduce the computational complexity of the conventional SLM PAPR reduction scheme, where the IFFT computations were reduced by generating a large number of the candidate signals from some of the remainder generated by IFFTs. In [7], Wang and Ouyang proposed the conversion matrices method to simplify the IFFT computations involved in the conventional SLM PAPR reduction scheme. Due to there only being two conversion matrices, the computational complexity of the conversion matrices method is only about half that of the conventional SLM PAPR reduction scheme. To further reduce the complexity, a large number of the conversion matrices were used to replace other IFFTs in [8]. With these conversion matrices, candidate signals were generated by only one or two IFFT outputs. The complexity in [8] is very low, but the BER performance is degraded due to the non-constant magnitudes of the frequency elements of the phase rotation vectors corresponding to the conversion matrices. In order to guarantee the BER performance of OFDM systems, new conversion matrices are designed in [9] based on the constant magnitudes of the frequency elements of the phase rotation vectors, but the proposed phase rotation vectors have a great correlation due to the periodic property, which leads to the performance loss of PAPR reduction. In [10], the candidate signals were generated by the linear combinations of the cyclically shifted interleaved partitions of the original OFDM signal. This method can achieve satisfactory PAPR reduction performance with greatly reduced computational complexity at the transmitter, but the receiver needs to detect and decode the transmitted side information (SI) with high computational complexity. However, all of the above SLM PAPR reduction schemes need the transmission of SI at the transmitter and perform the detection and decoding of SI at the receiver for correct data demodulation. In practice, the transmission of SI must be protected at the transmitter by using the channel coding method due to the frequency selective fading channel, and it is very important to correctly detect and decode the SI at the receiver since an erroneous detection of the SI will corrupt the entire received OFDM signal. This will further reduce the system throughput and increase the computational complexity at both the transmitter and receiver. In addition, there are some blind SLM PAPR reduction schemes [11-14] which do not need the transmission of SI. Unfortunately, they have huge complexity at both the transmitter and receiver and are not applicable in practical OFDM systems.

In this paper, we present a low-complexity blind SLM PAPR reduction scheme for OFDM signals. The proposed method is mainly focused on reducing the computational complexity of the SLM PAPR reduction scheme while avoiding the transmission of SI. Based on deriving the general time domain form of the conventional SLM PAPR reduction method, the proposed method properly designs the phase conversion vectors in the time domain at the transmitter as well as using the equivalent channel to perform the frequency domain equalization (FDE) at the receiver without SI transmission. As compared to the conversion vector-based SLM PAPR reduction method in [10], the proposed scheme has low computational complexity in PAPR reduction without the transmission of SI and obtains great robustness of BER performance.

The rest of this paper is organized as follows. The OFDM system model is introduced in Section 2. The new blind SLM PAPR reduction method is presented based on the proposed phase conversion vectors in the time domain and the computational complexity is analyzed in Section 3. The simulation results to illustrate the performance of the proposed scheme are presented in Section 4. Finally, the conclusions are drawn in Section 5. 


\section{System Models}

Let $\mathbf{X}=[X(0), X(1), \cdots, X(N-1)]^{\mathrm{T}}$ denote an input OFDM symbol block where $X(k)$ for $0 \leq k \leq N-1$ is the complex data transmitted at the $k$ th subcarrier and $N$ is the total number of subcarriers of OFDM systems. In the OFDM block, there are $N_{p}$ equally spaced pilot subcarriers for the purpose of channel estimation. By performing the $N$-point IFFT operation of $\mathbf{X}$, the time domain signal vector $\mathbf{x}=[x(0), x(1), \cdots, x(N-1)]^{\mathrm{T}}$ is obtained, which can be represented as

$$
\mathbf{x}=\mathbf{F X}
$$

where $\mathbf{F}$ is the $N \times N$ IFFT matrix whose element is $e^{j 2 \pi n k / N} / \sqrt{N}$ for $0 \leq n \leq N-1$ and $0 \leq k \leq N-1$. The $n$th element of $\mathbf{x}$ can be given as

$$
x(n)=\frac{1}{\sqrt{N}} \sum_{k=0}^{N-1} X(k) e^{j 2 \pi k n / N}, \quad 0 \leq n \leq N-1
$$

The PAPR of the transmitted OFDM signal vector $\mathbf{x}$ in Equation (1) can be defined as

$$
\operatorname{PAPR}(\mathbf{x})=\frac{\max _{0 \leq n \leq N-1}|x(n)|^{2}}{E\left[|x(n)|^{2}\right]}
$$

where $E[\cdot]$ denotes the expectation operation.

In the conventional SLM PAPR scheme, the phase rotation vectors can be described as $\mathbf{P}^{(u)}=\left[P^{(u)}(0), P^{(u)}(1), \cdots, P^{(u)}(N-1)\right]^{\mathrm{T}}$ for $0 \leq u \leq U-1$, where the $k$ th element of $\mathbf{P}^{(u)}$ is $P^{(u)}(k)=e^{j \theta^{(u)}(k)}$ with $\theta^{(u)}(k) \in(-\pi, \pi]$. Then, the $u$ th candidate signal in the frequency domain is generated by performing carrier-wise multiplication of the data vector $\mathbf{X}$ and the $u$ th phase rotation vector $\mathbf{P}^{(u)}$, and which can be given as

$$
\mathbf{X}^{(u)}=\left[P^{(u)}(0) X(0), \cdots, P^{(u)}(N-1) X(N-1)\right]^{\mathrm{T}}=\mathbf{Q}^{(u)} \mathbf{X}
$$

where $\mathbf{Q}^{(u)}=\operatorname{diag}\left\{\mathbf{P}^{(u)}\right\}$ is a diagonal matrix whose diagonal element is $P^{(u)}(k)$ for $k=0, \cdots, N-1$.

For better approximating the true PAPR in the discrete time case, usually $G$ times oversampling is considered. It was shown in [15] that $G=4$ is sufficient to capture the peak information. To obtain the oversampling vector $\mathbf{x}$ in the time domain, $N(G-1)$ zeros should be inserted in the middle of the input data vector $\mathbf{X}$ to form a $G N$-dimensional data vector given by $[X(0), \cdots, X(N / 2-1), 0, \cdots 0, X(N / 2), \cdots, X(N-1)]^{\mathrm{T}}$. Obviously, if $G$ times oversampling is considered, $\mathbf{F}$ is of $G N \times G N$ dimensions and $\mathbf{P}^{(u)}$ is of $G N$-dimensional phase rotation vector with $\mathbf{P}^{(u)}=\left[P^{(u)}(0), \cdots, P^{(u)}(N / 2-1), C^{(u)}(0), \cdots C^{(u)}((G-1) N-1), P^{(u)}(N / 2), \cdots, P^{(u)}(N-1)\right]^{\mathrm{T}}$ where $C^{(u)}(k)$ can be an arbitrary value for $k=0, \cdots,(G-1) N-1$. Finally, the $u$ th candidate signal vectors $\mathbf{x}^{(u)}=\left[x^{(u)}(0), x^{(u)}(1), \cdots, x^{(u)}(G N-1)\right]^{\mathrm{T}}$ in the time-domain can be obtained and which can be expressed as

$$
\mathbf{x}^{(u)}=\mathbf{F} \mathbf{X}^{(u)}=\mathbf{F}\left(\mathbf{Q}^{(u)} \mathbf{X}\right)
$$

Among these candidate signals $\mathbf{x}^{(u)}$ for $0 \leq u \leq U-1$, the one with the lowest PAPR is selected to be transmitted, which can be given as follows

$$
\mathbf{x}^{(\bar{u})}=\underset{\mathbf{x}^{(u)}, 0 \leq u \leq U-1}{\operatorname{argmin}} \operatorname{PAPR}\left(\mathbf{x}^{(u)}\right)
$$

To avoid the inter-signal interference caused by the wireless channel, a cyclic prefix $(\mathrm{CP})$ is inserted at the beginning of each transmitted signal whose length is always assumed to be larger than that 
of the wireless channel. At the receiver, the received discrete time domain signal can be expressed as follows

$$
y(n)=\sum_{m=0}^{L-1} h(m) x^{(\bar{u})}((n-m))_{N}+w(n), 0 \leq n \leq N-1
$$

where $\mathbf{y}=[y(0), y(1), \cdots, y(N-1)]^{\mathrm{T}}$ is the received signal vector, $((\cdot))_{N}$ denotes a cyclic shift in the base of $N, \mathbf{h}=[h(0), h(1), \cdots, h(L-1)]^{\mathrm{T}}$ is the impulse response in the time domain of the wireless channel, $L$ is the total number of resolvable multi-paths of the wireless channel and $\mathbf{w}=[w(0), w(1), \cdots, w(N-1)]^{\mathrm{T}}$ represents the additive white Gaussian noise (AWGN) vector. Then, after performing a fast Fourier transform (FFT) of $\mathbf{y}$, the received frequency domain data $\mathbf{Y}=[Y(0), Y(1), \cdots, Y(n-1)]^{T}$ can be expressed as

$$
\mathbf{Y}=\mathbf{F}^{H} \mathbf{y}
$$

Therefore, the element on the $k$ th subcarrier is

$$
Y(k)=H(k) P^{(\bar{u})}(k) X(k)+W(k), 0 \leq k \leq N-1
$$

where $W(k)$ is the FFT of the noise sequence $w(n)$ and $H(k)$ denotes the channel frequency response on the $k$ th subcarrier, which is given by

$$
H(k)=\sum_{l=0}^{L-1} h(l) e^{-j \frac{2 \pi k l}{N}}
$$

As we know, the pilot tones can be used to estimate the frequency response of the fading channel [14]. Based on the estimated channel, FDE can be performed on the received data, and then transmitted data can be recovered from the received data.

\section{Proposed SLM PAPR Scheme}

In this section, a novel low-complexity SLM scheme without the transmission of SI is proposed to reduce the PAPR of OFDM signals. The proposed method properly designs the time domain conversion vectors based on the equivalent wireless channel and the transmitter does not transmit the SI. At the receiver, the effect of the carrier-wise multiplication of the phase rotation vector and the data vector can be removed by using the conventional channel equalization method. Also, it is shown that the proposed scheme has very low computational complexity.

\subsection{Derived General Form of SLM in the Time Domain}

From Equation (4), according to the circular convolution property, the $n$th element of candidate signal vectors $\mathbf{x}^{(u)}$ can be given as follows:

$$
x^{(u)}(n)=\sum_{m=0}^{N-1} p^{(u)}(m) x((n-m))_{N}, 0 \leq n \leq N-1
$$

where $p^{(u)}(m)$ is the $m$ th element of $\mathbf{p}^{(u)}=\mathbf{F} \mathbf{P}^{(u)}$.

By comparing Equations (7) and (8), it can be seen that the generated time domain candidate signals of the SLM scheme is equivalent to passing the original signal through a tap-delayed wireless channel. Figure 1 shows the equivalent structure in generating candidate signals of the SLM scheme in the time domain. As shown in Figure 1, the parameter of the related channel in the SLM scheme depends on the used phase rotation vector $\mathbf{P}^{(u)}$ and can be given as

$$
p^{(u)}(n)=\sum_{l=0}^{N-1} p^{(u)}(l) \delta(n-l), 0 \leq n \leq N-1
$$


where $\delta(\cdot)$ denotes the Dirac-delta function.

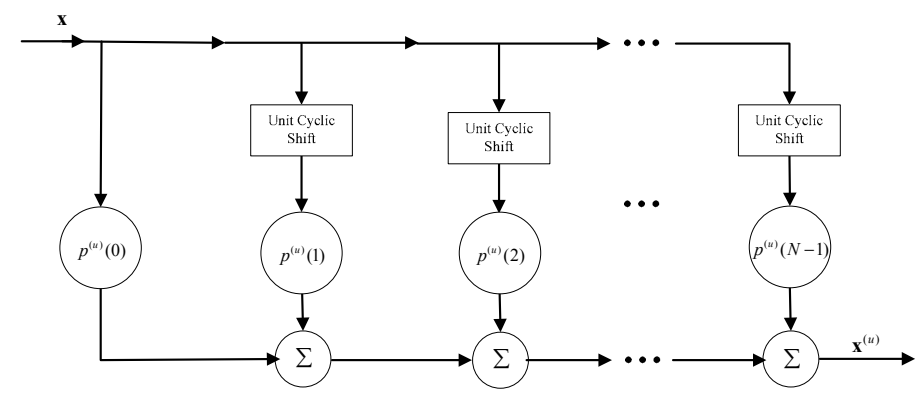

Figure 1. Equivalent structure of SLM in generating candidate signals in the time domain.

It should be pointed out that the wireless channel parameter characterized by Equation (12) does not exist in most practical cases due to its length $N$ if $\mathbf{p}^{(u)}=\left[p^{(u)}(0), p^{(u)}(1), \cdots, p^{(u)}(N-1)\right]^{\mathrm{T}}$ has relatively small support, i.e., $p^{(u)}(n)=0$ for a certain small $n$. Let the support of $\mathbf{p}^{(u)}$ be denoted as $L_{u}$ and $\mathbf{p}^{(u)^{\prime}}=\left[p^{(u)}(0), p^{(u)}(1), \cdots, p^{(u)}\left(L_{u}-1\right)\right]^{\mathrm{T}}$. If $L_{u}+L-1$ is smaller than the length of CP, the general form of the SLM scheme in the time domain can be described by Figure 2.

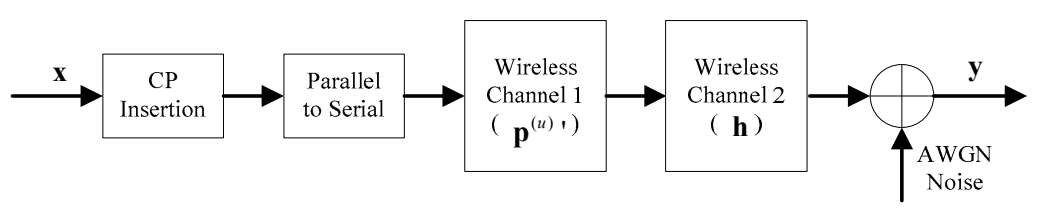

Figure 2. General form of selected mapping (SLM) scheme in the time domain.

As shown in Figure 2, the final equivalent channel of the SLM scheme can be given as

$$
\mathbf{h}_{e q}=\mathbf{p}^{(u)^{\prime}} \otimes \mathbf{h}
$$

where $\otimes$ is the circular convolution operator. The channel frequency response of $\mathbf{h}_{\ell q}$ can be given as

$$
\mathbf{H}_{e q}=\mathbf{Q}^{(u)} \mathbf{H}
$$

where $\mathbf{H}=[H(0), H(1), \ldots, H(N-1)]^{\mathrm{T}}$ is the original channel frequency response vector.

According to Equation (13), when the support of $\mathbf{h}_{e q}$ is smaller than the number of pilot subcarriers in every OFDM symbol, $\mathbf{h}_{e q}$ can be estimated in the same way as $\mathbf{h}$. In the following, it will be seen that the general form of the SLM scheme in the time domain can be used to realize low complexity without SI transmission.

\subsection{Proposed Low-Complexity Conversion Vectors}

As with the proposed SLM PAPR scheme in [10], the IFFT operation is replaced by the conversion vectors $T_{i}$ for $i=1, \cdots, U$ and the candidate OFDM signals are generated by performing circular convolution of the IFFT of the original OFDM symbol block with the conversion vectors. The basic architecture of the SLM PAPR reduction scheme using conversion vectors in [10] is shown in Figure 3. It was indicated that the computational complexity at the transmitter can be greatly reduced, and each conversion vector requires only $3 \mathrm{~N}$ complex additions. 


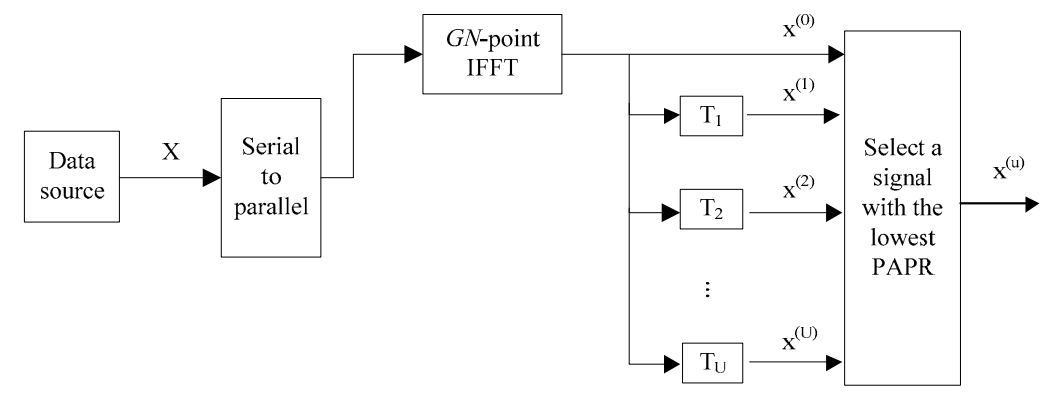

Figure 3. Basic architecture of the SLM scheme using conversion vectors.

From the previous discussion, we know that when the conversion vectors are properly designed in the time domain, conversion vectors can be an equivalent channel and the effect of the conversion matrix can be removed by the channel equalization operation. Based on the conversion vector structure, in order to implement the low-complexity SLM scheme without SI, the proposed conversion vector $\mathbf{p}^{(u)}$ can be designed according to the following rules:

(1) The support $L_{u}$ of $\mathbf{p}^{(u)}$ is chosen as a small integer to restrict the length of the equivalent channel;

(2) There are few nonzero elements whose number is denoted as $K$ in $\mathbf{p}^{(u)}$ to reduce the complexity in generating the candidate signals with Equation (11);

(3) The real and imaginary parts of each nonzero element in $\mathbf{p}^{(u)}$ are chosen in the set $\{0, \pm 1\}$ to avoid the complex multiplications in generating the candidate signals (ignoring the constant factor to maintain the power constraint) [10].

Generally speaking, there are many choices for the exact values of $L_{u}$ and $K$. The support $L_{u}$ in constraint (1) and the number $K$ may vary depending on the application. Therefore, the proposed scheme can achieve a flexible compromise between computational complexity and BER performance. It can be used for any system with the parameters chosen according to the application. For example, to have comparable complexity with the available works, the values of $K$ can be selected as $K=3$ or $K=4$ and the values of $L_{u}$ can be selected as smaller than eight samples. Given the parameters for $K$ and $L_{u}$, we can properly design the vectors $\mathbf{p}^{(u)}$ to generate the candidate signals with low-complexity without the transmission of SI. To achieve good BER performance, we can design the vectors $\mathbf{p}^{(u)}$ with the following two steps.

In the first step, we construct a set $\Re=\left\{\mathbf{r}^{(1)}, \mathbf{r}^{(2)} \ldots, \mathbf{r}^{(V)}\right\}$ with $V(V$ can be much larger than $U)$ vectors according to the above three rules for the given values of $K$ and $L_{u}$ by varying the values and positions of the nonzero elements in $\mathbf{r}^{(i)}, 1 \leq i \leq V$. One example of generating $\Re$ used in this paper is shown in Table 1 , where $\mathbf{T}=\left\{T_{0}, T_{1}, \ldots, T_{K-1}\right\}\left(T_{K}=L_{u}-1\right)$ is the set of the indices of the nonzero elements and $K$ is the number of nonzero elements in $\mathbf{r}^{(i)}, 1 \leq i \leq V$, and $\mathbf{a}=\left\{a^{(0)}, a^{(1)}, \ldots, a^{(K-1)}\right\}$ is the set of the values of the nonzero elements. Clearly, given the parameters of $K$ and $L_{u}$, a large number of vectors $\mathbf{r}^{(i)}$ can be generated.

Table 1. Parameters chosen for generating candidate vectors.

\begin{tabular}{ccc}
\hline $\begin{array}{c}\text { Number of Nonzero } \\
\text { Elements }\end{array}$ & Indices of Nonzero Elements & Values of Nonzero Elements \\
\hline$K=3$ & $\mathbf{T}=\{0,3,6\}, \mathbf{T}=\{0,4,5\}$, & $a^{(0)}=1, a^{(1)}, a^{(2)}=\{ \pm 1, \pm j\}$ and \\
and $\mathbf{T}=\{0,2,7\}$ & $a^{(0)}=1-j, a^{(1)}, a^{(2)}=\{ \pm 1, \pm j\}$ \\
$K=4$ & $\mathbf{T}=\{0,5,6,7\}$ and $\mathbf{T}=\{0,3,7,8\}$ & $a^{(0)}=1, a^{(1)}, a^{(2)}, a^{(3)}=\{ \pm 1, \pm j\}$ \\
\hline
\end{tabular}

In the second step, we take the FFT of the $V$ vectors in $\Re$ to generate $V$ phase rotation vectors. Then, we choose $U-1$ phase rotation vectors from them such that the smallest amplitude 
of the elements of each selected phase rotation vector is larger than that of the other vectors. The corresponding vectors of the selected $U-1$ phase rotation vectors in $\Re$ results in the final set $\Re^{\prime}=\left\{\mathbf{p}^{(0)}, \ldots, \mathbf{p}^{(i)}, \mathbf{p}^{(U-1)} \mid \mathbf{p}^{(i)} \in \Re\right\}$, where $\mathbf{p}^{(0)}=[1,0, \ldots, 0]^{\mathrm{T}}$ represents the vector corresponding to the original signal. Then, with the final set $\Re^{\prime}$, the candidate signals can be generated by using Equation (11).

Since the equivalent channel can be expressed by Equation (10), it is easy to see that the length of the equivalent channel is $L^{\prime}=L_{u}+L-1$. Due to the small values of $L_{u}, L^{\prime}$ can also be smaller than the number of pilot subcarriers used for channel estimation in the OFDM system. Therefore, the equivalent channel in the proposed SLM scheme can be estimated in the same way as that in the conventional OFDM system, and the effect of the applied phase rotation vector can be removed in the equalization procedure. In addition, the coherent bandwidth of the equivalent may be slightly smaller than that of the conventional OFDM system. It is noted that, in many practical OFDM systems, the spacing between two consecutive pilot sub-channels is sufficiently small as compared to the coherent bandwidth of the wireless channel. In such systems, the channel frequency responses can be obtained simply by linear interpolation between the channel frequency responses at two consecutive pilot sub-channels.

Remark 1. In the proposed scheme, by making full use of the equivalent channel, the proposed SLM scheme can easily equalize the received data in the frequency domain as the conventional OFDM systems without SI detection and decoding. This does not increase the computational load at the receiver.

Remark 2. It is pointed out in [16] that only the simplified SLM scheme [17] can be used in the point-to-multipoint scenarios. However, by using the equivalent channel, the proposed SLM scheme can be easily extended to the multiple-input multiple-output (MIMO) systems without special signal processing algorithms at both the transmitter and receiver.

\subsection{Complexity Analysis}

The computational complexity of the proposed PAPR reduction method can be evaluated in terms of the number of complex multiplications (CMs) and complex additions (CAs) required for OFDM systems. For an OFDM system with $N$ subcarriers and $G$ times oversampling, each IDFT operation requires (GN/2) $\log _{2}(G N) \mathrm{CMs}$ and $G N \log _{2}(G N) \mathrm{CAs}$, respectively.

In the conventional SLM PAPR method, the transmitter requires $U$ IDFT operations to generate $U$ candidate signals. Hence, the conventional SLM method requires $(U G N / 2) \log _{2}(G N)$ CMs and UGN $\log _{2}(G N)$ CAs.

At the transmitter, both the proposed method and the SLM Method in [10] use a conversion matrix to generate $(U-1)$ different candidate signals after one IDFT operation, and each conversion matrix only has the number of $K$ nonzero elements. Thus, the computational complexity of the transmitter is the same for both methods, and the transmitter requires $(G N / 2) \log _{2}(G N) C M s$ and $G N \log _{2}(G N)+$ $(U-1)(K-1) G N$ CAs.

Table 2 summarizes the computational complexities of the three PAPR schemes at the transmitter. It is seen that the conventional SLM method requires the greatest number of CMs and CAs of the three methods. Moreover, it is observed that the SLM scheme in [10] and the proposed method require the same number of CMs and CAs at the transmitter.

Table 2. Comparisons of computational complexity at the transmitter. PAPR: peak-to-average power ratio; CM: complex multiplications; CA: complex additions.

\begin{tabular}{ccc}
\hline PAPR Schemes & Number of CMs & Number of CAs \\
\hline Conventional SLM method & $(U G N / 2) \log _{2}(G N)$ & $U G N \log _{2}(G N)$ \\
SLM method in [10] & $(G N / 2) \log _{2}(G N)$ & $G N \log _{2}(G N)+(U-1)(K-1) G N$ \\
Proposed method & $(G N / 2) \log _{2}(G N)$ & $G N \log _{2}(G N)+(U-1)(K-1) G N$ \\
\hline
\end{tabular}


The above discussion gives the comparisons of the computational complexity of the three methods at the transmitter. It should be noted that, as with the conventional SLM method, the SLM method in [10] needs to transmit SI to indicate which conversion matrix is chosen. If $U$ candidate signals are generated, $\log _{2}(U) \mathrm{SI}$ bits are required for the receiver to recover the original transmitter signal. At the receiver, the decision procedure of SI still requires a number of computational complexities for the SLM method in [10]. However, the proposed method without SI transmission does not need additional computational complexity and the effect of the conversion matrix can be removed by the channel equalization operation. Obviously, the receiver of the SLM scheme in [10] requires significantly higher computational complexity than that of the proposed method. Thus, the overall computational complexity of the proposed method is the lowest in the three schemes at the transmitter and the receiver.

\section{Simulation Results}

To perform numerical analysis, computer simulations are conducted to verify the performance of the proposed modified SLM application with the derived general form (denoted as G-MSLM). For the purpose of comparison, the SLM scheme in [10] is also considered in our simulations. The considered OFDM system has total subcarriers $N=256$ with 16 QAM modulation and its bandwidth is $20 \mathrm{MHz}$. The pilot subcarriers are equally distributed every six subcarriers. To evaluate the performance of PAPR reduction, the oversampling factor is specified as $G=4$. The parameters in generating $\Re$ is selected as $K=3, L_{u}=7$ with the set of the indices of the nonzero elements $\mathbf{T}=\{0,3,6\}, \mathbf{T}=\{0,4,5\}$, and $\mathbf{T}=\{0,2,7\}$, and $K=3, L_{u}=8$ with $\mathbf{T}=\{0,5,6,7\}$ and $\mathbf{T}=\{0,3,7,8\}$.

Figure 4 shows a comparison of PAPR reduction performance between G-MSLM and the SLM scheme in [10] in terms of complementary cumulative distribution function (CCDF). From this figure, we can see that, for $M=16, M=32$ and $M=57$, respectively, G-MSLM can achieve almost the same PAPR reduction performance as that of the SLM scheme in [10]. However, it is noted that the G-MSLM scheme can avoid the transmission of SI, and therefore it has the potential of using more candidate signals without reducing the transmission data rate, such as the case for $M=114$. As compared to the original OFDM systems, the gains in PAPR of G-MSLM are $3.7 \mathrm{~dB}$ and $4.2 \mathrm{~dB}$, respectively, for $M=57$ and $\operatorname{Pr}\left[\mathrm{PAPR} \geq \mathrm{PAPR}_{0}\right]=10^{-3}$ and $\operatorname{Pr}\left[\mathrm{PAPR} \geq \mathrm{PAPR}_{0}\right]=10^{-4}$.

Figure 5 shows the BER performance of G-MSLM in wireless channels with the exponential-decay power-delay profile (PDF) without considering the nonlinearity of HPA. From this figure, it can be seen that the BER performance of G-MSLM becomes slightly worse with the increase of $M$. In addition, there is a small BER performance loss comparing the G-MSLM with the original OFDM signal. Since the length of the equivalent channel is slightly larger than that of the real wireless channel, the proposed G-MSLM method will increase the frequency selectivity of the compared with that of the original wireless channel.

Figure 6 shows the BER performance of G-MSLM in the same channel as that in Figure 5 considering the nonlinearity of HPA. To take the nonlinear HPA into account at the transmitter, the amplitude of transmitted signal is adjusted (with the peak amplitude normalized to be 1 as that in [18]), in which case nonlinear distortions will rarely occur. From this figure, we can see that G-MSLM can achieve better BER performance than the original OFDM system. It has about $3 \mathrm{~dB}$ improvement over the original OFDM system. The improvement of G-MSLM over the original OFDM system is due to the fact that G-MSLM can achieve much lower PAPR, which allows a higher level of the transmitted signal. In addition, it can be seen that the BER performance of G-MSLM for $M=114$ is slightly better than that for $M=57$ due to its better PAPR reduction performance. Note that since the transmitted signals are normalized with peak amplitude to be 1 , the $x$-axis is $1 / N_{0}$ where $N_{0}$ is the single-sided power spectral density of the additive white noise. 


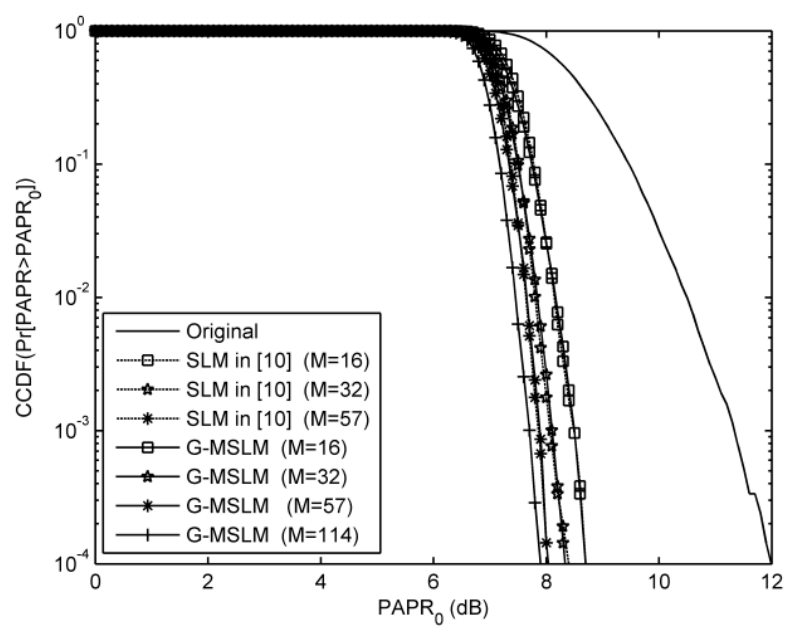

Figure 4. PAPR reduction performance comparisons. G-MSLM: derived general form of modified SLM.

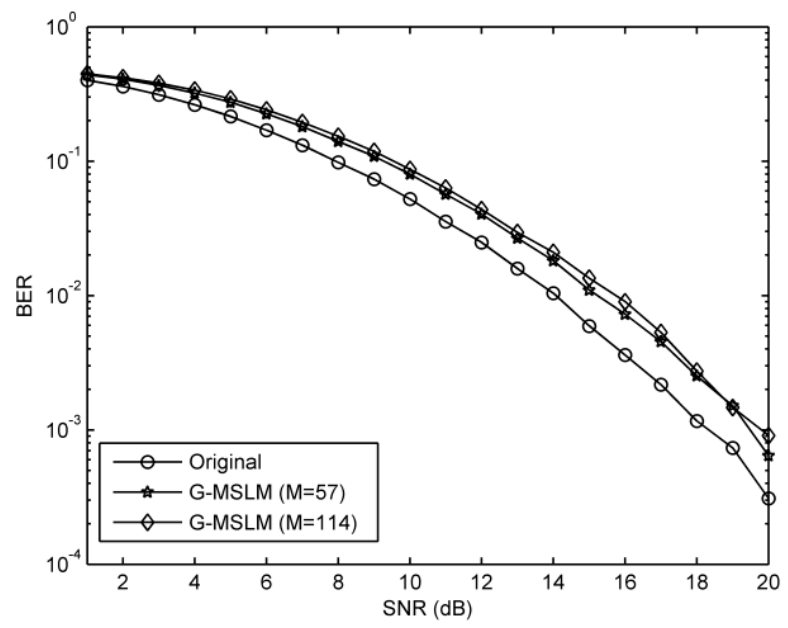

Figure 5. BER performance comparisons under multipath fading channels.

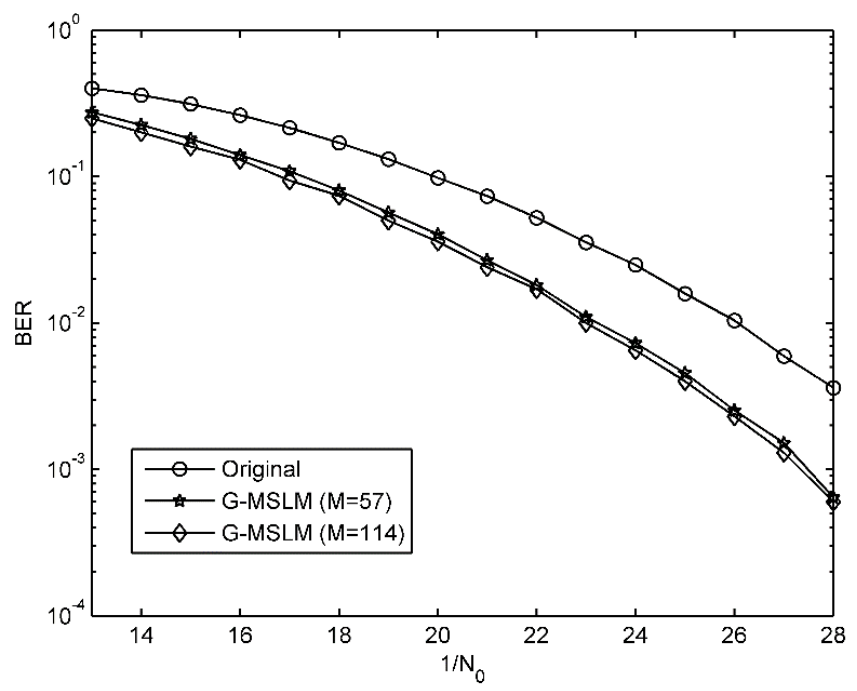

Figure 6. Bit error rate (BER) performance comparisons with nonlinear high-power amplifiers (HPA). 


\section{Conclusions}

In this paper, novel phase rotation vectors are designed based on deriving the general form of a conventional SLM PAPR reduction scheme in the time domain, and then a novel blind SLM PAPR reduction method is proposed with low complexity. At the transmitter, the phase rotation vector can be considered as an equivalent wireless channel without SI transmission. At the receiver side, it does not need to detect the SI and the effect of phase rotation vectors can be removed by a conventional channel estimation method. Therefore, the proposed scheme has the advantage of being transparent to the receiver, which avoids the transmission of SI and does not increase the data demodulation complexity of the convectional OFDM system. In addition, the proposed scheme has the flexibility of choosing parameters according to practical application. Since the proposed scheme can achieve good PAPR reduction performance without the transmission of SI, the proposed PAPR reduction scheme can be also used in other applications such as the single-carrier frequency-division multiple access (SC-FDMA) system [19] and OFDM-based wavelength division multiplexing (WDM) systems [20,21].

Author Contributions: Y.X. and J.J. proposed the low-complexity SLM-based PAPR reduction method and formal analysis; Y.X. provided the simulation and comparative analysis; Y.X. and J.J. wrote the paper together.

Acknowledgments: This work was supported by the Key Scientific Research Program of Higher Education of Henan Province of China under Grant No. 17A510013.

Conflicts of Interest: The authors declare no conflict of interest.

\section{References}

1. Jiang, T.; Wu, Y. An overview: peak-to-average power ratio reduction techniques for OFDM signals. IEEE Trans. Broadcast. 2008, 54, 257-268. [CrossRef]

2. Hasan, M. A novel CVM precoding scheme for PAPR reduction in OFDM transmissions. Wirel. Netw. 2014, 20, 1573-1581. [CrossRef]

3. Adegbite, S.A.; McMeekin, S.G.; Stewart, B.G. A joint OFDM PAPR reduction and data decoding scheme with no SI estimation. EURASIP J. Wirel. Commun. Netw. 2016, 108, 1-12. [CrossRef]

4. Sohn, I.; Chul, S. Neural network based simplified clipping and filtering technique for PAPR reduction of OFDM signals. IEEE Commun. Lett. 2015, 19, 1438-1441. [CrossRef]

5. Mazahir, S.; Amin, S. On companding schemes for PAPR reduction in OFDM systems employing higher order QAM. IEEE Trans. Broadcast. 2016, 62, 716-726. [CrossRef]

6. Heo, S.; Noh, H.; No, J.; Shin, D. A modified SLM scheme with low complexity for PAPR reduction of OFDM systems. IEEE Trans. Broadcast. 2007, 53, 804-808. [CrossRef]

7. Wang, C.; Hsu, M.; Ouyang, Y. A low-complexity peak-to-average power ratio reduction technique for OFDM systems. In Proceedings of the 2003 Global Telecommunications Conference, San Francisco, CA, USA, 1-5 December 2003.

8. Wang, C.; Hsu, M.; Ouyang, Y. Low-complexity selected mapping schemes for peak-to-average power ratio reduction in OFDM systems. IEEE Trans. Signal Process. 2005, 53, 4652-4660. [CrossRef]

9. Wang, C.; Ku, S. Novel conversion matrices for simplifying the IFFT computation of an SLM-based PAPR reduction scheme for OFDM systems. IEEE Trans. Wirel. Commun. 2009, 57, 1903-1907. [CrossRef]

10. Li, C.; Wang, S.; Wang, C. Novel low-complexity SLM schemes for PAPR reduction in OFDM systems. IEEE Trans. Signal Process. 2010, 58, 2916-2921. [CrossRef]

11. Jayalath, A.; Tellambura, C. SLM and PTS peak-power reduction of OFDM signals without side information. IEEE Trans. Wirel. Commun. 2005, 4, 2006-2013. [CrossRef]

12. Joo, H.; Heo, S.; Jeon, H.; No, J.; Shin, D. A new blind SLM scheme with low decoding complexity for OFDM systems. IEEE Trans. Broadcast. 2012, 58, 669-676. [CrossRef]

13. Goff, S.; Al-Samahi, S.; Khoo, B.; Tsimenidis, C.; Sharif, B. Selected mapping without side information for PAPR reduction in OFDM. IEEE Trans. Wirel. Commun. 2009, 8, 3320-3325. [CrossRef]

14. Park, J.; Hong, E.; Har, D. Low complexity data decoding for SLM based OFDM systems without side information. IEEE Commun. Lett. 2011, 15, 611-613. [CrossRef] 
15. Tellambura, C. Computation of the continuous-time PAR of an OFDM signal with BPSK subcarriers. IEEE Commun. Lett. 2001, 5, 185-187. [CrossRef]

16. Gerhard, W.; Robert, F.; Holge, B. The PAPR problem in OFDM transmission: new directions for a long-lasting problem. IEEE Signal Process. Mag. 2013, 30, 130-144. [CrossRef]

17. Baek, M.; Kim, M.; You, Y.; Song, H. Semi-blind channel estimation and par reduction for MIMO-OFDM system with multiple antennas. IEEE Trans. Broadcast. 2004, 50, 414-424. [CrossRef]

18. Yuen, C.; Behrouz, F. Analysis of the optimum precoder in SC-FDMA. IEEE Trans. Wirel. Commun. 2012, 11, 4096-4107. [CrossRef]

19. Tsiropoulou, E.E.; Kapoukakis, A.; Papavassiliou, S. Uplink resource allocation in SC-FDMA wireless networks: A survey and taxonomy. Comput. Netw. 2016, 96, 1-28. [CrossRef]

20. Malka, D.; Zalevsky, Z.; Sintov, Y. Design of a $1 \times 4$ silicon wavelength demultiplexer based on multimode interference in a slot waveguide structures. J. Opt. 2015, 17, 1-9. [CrossRef]

21. Ben Zaken, B.B.; Zanzury, T.; Malka, D. An 8-channel wavelength mmi demultiplexer in slot waveguide structures. Materials 2016, 9, 881. [CrossRef] [PubMed]

(C) 2018 by the authors. Licensee MDPI, Basel, Switzerland. This article is an open access article distributed under the terms and conditions of the Creative Commons Attribution (CC BY) license (http:// creativecommons.org/licenses/by/4.0/). 